\title{
POLÍTICAS DE MEDICAMENTOS, ASSISTÊNCIA FARMACÊUTICA E VIGILÂNCIA SANITÁRIA NO BRASIL: ANÁLISE DA ATUAÇÃO DO ESTADO NO PERÍODO DE 2014 A 2018
}

\author{
MEDICATION POLICY, PHARMACEUTICAL ASSISTANCE AND \\ SANITARY SURVEILLANCE IN BRAZIL: ANALYSIS OF THE STATE'S \\ PERFORMANCE IN THE PERIOD 2014 TO 2018
}

\author{
Mariluce Karla Bomfim de Souza ${ }^{1}$ \\ Yara Oyram Ramos Lima ${ }^{2}$ \\ Patricia Sodré Araújo ${ }^{3}$ \\ Ediná Alves Costa ${ }^{4}$ \\ Gisélia Santana Souza ${ }^{5}$ \\ Ana Cristina Souto ${ }^{6}$
}

\begin{abstract}
Resumo: O artigo trata da análise das políticas de medicamentos, assistência farmacêutica e vigilância sanitária, que constituem um dos eixos temáticos do Observatório de Análise Política em Saúde (OAPS), espaço caracterizado por uma rede de pesquisadores do Instituto de Saúde Coletiva da Universidade Federal da Bahia (ISC/UFBA) e outros centros de pesquisa. Assim, questiona-se como o Estado tem atuado e/ou se posicionado no que tange às políticas de Medicamentos e de Assistência Farmacêutica no período de 2014 à 2018? Tem por objetivo: apresentar os resultados da análise da atuação do Estado brasileiro em torno das referidas políticas, no período de 2014 a 2018, tendo em vista o uso de estratégias de pesquisa utilizadas a partir de notícias de sites institucionais. Os resultados apresentam-se em quatro categorias definidas pelo OAPS: implementação, financiamento, participação social e resultados da política. Em que pesem os avanços, o subfinanciamento público da saúde e os conflitos de interesse, entre outros elementos, oferecem ameaças, requerendo ação contínua do Estado e dos atores interessados na saúde como um direito social que inclui medicamentos, regulação e vigilância sanitária.
\end{abstract}

Palavras-chave: Política de Saúde; Medicamento; Assistência Farmacêutica; Vigilância Sanitária; Direito à Saúde; Participação Social.

\footnotetext{
Abstract: The article deals with the analysis of drug policy, pharmaceutical services policy and health surveillance policy, which are one of the thematic axes of the Observatory of Political Analysis in Health (OAPS), a space characterized by a network of researchers from the Institute of Collective Health of the Federal University of Bahia (ISC/UFBA) and other research centers. Thus, it is questioned how the State

${ }^{1}$ Pós Doutorado pela Universidad Complutense de Madrid, Espanha (UCM/ES). Professora da Universidade Federal da Bahia (UFBA), Salvador, Bahia, Brasil. E-mail: marilucejbv@ yahoo.com.br

${ }^{2}$ Doutorado em Saúde Pública pela Universidade Federal da Bahia (UFBA). Professora da Universidade Federal da Bahia (UFBA), Salvador, Bahia, Brasil. E-mail: oyram@ufba.br

${ }^{3}$ Doutorado em Saúde Pública pela Universidade Federal da Bahia (UFBA). Professora da Universidade do Estado da Bahia (UNEB), Salvador, Bahia, Brasil. E-mail: patrisodré@ gmail.com

${ }^{4}$ Doutorado em Saúde Pública pela Universidade de São Paulo (USP). Professora da Universidade Federal da Bahia (UFBA), Salvador, Bahia, Brasil. E-mail: edina@ufba.br

${ }^{5}$ Doutorado em Saúde Pública pela Universidade Federal da Bahia (UFBA). Professora da Universidade Federal da Bahia (UFBA), Salvador, Bahia, Brasil. E-mail: gsantanasouza@gmail.com

${ }^{6}$ Doutorado em Saúde Pública pela Universidade Federal da Bahia (UFBA). Professora da Universidade Federal da Bahia (UFBA), Salvador, Bahia, Brasil. E-mail: ana1 souto@ yahoo.com.br
} 
has acted and/or positioned itself regarding the policies of Medications and Pharmaceutical Services in the period from 2014 to 2018 ? The purpose of this study is to present the results of the analysis of the Brazilian State's actions around these policies in the period from 2014 to 2018, in view of the use of research strategies used from news from institutional sites. The results are presented in four categories defined by the OAPS: implementation, financing, social participation and policy results. Regarding advances, public underfunding of health and conflicts of interest, among other elements, offer threats, requiring continuous action by the State and stakeholders interested in health as a social right that includes medicines, regulation an health surveillance.

Keywords: Health Politics; Drug; Pharmaceutical Assistance; Health Surveillance; Right to Health; Social Participation.

\section{Introdução}

O tema medicamentos e questões relacionadas permanecem, historicamente, como objeto de debate internacional e de preocupações nos diversos países como um dos mais sensíveis nos sistemas de saúde em todo o mundo e está presente na Agenda Internacional do Desenvolvimento. A tecnologia mais utilizada nos serviços de saúde (BARROS, 2008a) e amplamente fora deles (BARROS, 2008b), o medicamento, considerado um dos mais eficientes instrumentos da terapêutica moderna (LEFÈVRE, 1991), revela-se um objeto complexo com múltiplas faces: possui benefícios e porta riscos, intrínsecos e potenciais; é um bem social e ao mesmo tempo uma mercadoria, que requer permanente atividade regulatória, dado que se encontra permeado por distintos interesses e muitas questões que afetam a saúde da coletividade e os sistemas de saúde (COSTA et al., 2017). Nos termos de Pignarre (1999), o medicamento é um objeto estranho entre ciência, mercado e sociedade. A disponibilidade de medicamentos para garantir o acesso a toda a população representa um grande desafio. Além de problemas relativos ao acesso, à qualidade e até mesmo à falsificação, existem problemas relacionados à sua utilização (BERMUDEZ; BARROS, 2016).

Nas últimas décadas, o Brasil experimentou importantes transformações de natureza econômica, social, política e demográfica, com alterações significativas no perfil epidemiológico, em consequência da industrialização e urbanização, dos avanços sociais e do envelhecimento da população (VIACAVA et al., 2018). Entretanto, permanecem significativos contingentes populacionais em situação de pobreza e más condições de vida, o que resulta na manutenção de problemas de saúde já superados ou controlados em países desenvolvidos (RIBEIRO, 2014).

Com o Sistema Único de Saúde (SUS), criado com a Constituição de 1988 no processo de redemocratização do país, a atenção à saúde da população tem passado por importantes mudanças: a ampliação da oferta de serviços e do acesso, mudanças nos 
padrões de utilização e crescimento do número de profissionais vinculados ao SUS, entre outras. Contudo, permanecem desafios históricos tais como marcantes desigualdades regionais, subfinanciamento e uma complexa relação público-privado na prestação dos serviços de saúde, à medida que a garantia do acesso aos serviços públicos de saúde para a população requer a contratação de serviços privados e muitos destes também dependem de recursos públicos para sua manutenção e expansão. Acrescente-se o mais recente desafio representado pelos prováveis efeitos negativos decorrentes do congelamento dos gastos sociais por 20 anos, a partir de 2016 (VIACAVA et al., 2018).

Decorridos 30 anos do SUS, Paim (2018, p. 1724) sinaliza "vetores positivos" que contribuíram para a sustentabilidade do SUS, dentre eles a "engenharia políticoinstitucional criativa" que possibilitou a operacionalização de um conjunto de serviços e a disposição de equipamentos e tecnologias. Nesse conjunto, tais políticas têm destaque pelos seus avanços, a assistência farmacêutica, a vigilância sanitária e os hemocentros, dentre outros, destacados como "nichos" ou territórios de excelência (SANTOS, 2018a), de modo que a política do sangue e seu controle de qualidade, bem como a qualidade dos registros de medicamentos e demais produtos (SANTOS, 2018b) têm respeito no âmbito internacional.

O reconhecimento, no Brasil, do direito à saúde como direito humano e universal assegurou o acesso aos medicamentos pelo SUS, que deve prover, como responsabilidade do Estado, a assistência farmacêutica, que abrange o acesso a medicamentos de qualidade com os necessários cuidados farmacêuticos e um conjunto de ações dirigidas aos prescritores, profissionais de saúde, cidadãos e ao segmento produtivo farmacêutico para que os medicamentos sejam de qualidade, eficazes e seguros, com acesso regular aos que deles necessitam e utilizados segundo o princípio do uso racional (COSTA et al., 2017).

As políticas farmacêuticas estabelecidas com a Política Nacional de Medicamentos - PNM (BRASIL, 1998), a Política de Medicamentos Genéricos (BRASIL, 1999a), a Política Nacional de Assistência Farmacêutica - PNAF (BRASIL, 2004) e dispositivos da Lei $n^{\circ}$ 9872/1999 (BRASIL, 1999b), que criou a Agência Nacional de Vigilância Sanitária (Anvisa), entre outras políticas específicas, são acompanhadas seguidamente por diversas ações, desencadeadas, principalmente pela PNM e pela PNAF, e pela elaboração e operacionalização de normas, portarias, resoluções, decretos e outros dispositivos para a regulação e vigilância sanitária dos medicamentos, a organização e o financiamento da assistência farmacêutica, o acesso 
aos medicamentos, a organização e a gestão dos serviços farmacêuticos nas distintas esferas de governo.

Na Pesquisa Nacional sobre Acesso, Utilização e Promoção do Uso Racional de Medicamentos - PNAUM 2016, 94,3\% dos entrevistados no inquérito declararam ter tido acesso total aos medicamentos para as DCNT analisadas, e o acesso total a esses medicamentos foi maior entre indivíduos com mais de 60 anos (OLIVEIRA et al., 2016). Contudo, desafios persistem, pois os resultados demonstraram padrões de desigualdades no acesso entre as regiões do país (OLIVEIRA et al., 2016), bem como no processo de institucionalização da assistência farmacêutica (SOUZA et al., 2017), na situação sanitária dos medicamentos (COSTA et al., 2017) e na oferta de serviços farmacêuticos clínicos (ARAÚJO et al., 2017). Ressalta-se que nem todos os países desenvolvidos que possuem sistemas de saúde universais asseguram acesso universal e integral aos medicamentos (MORGAN; BOOTHE, 2016). No Brasil, em que pesem os desafios, o acesso universal e integral aos medicamentos no SUS é um direito social (LUIZA, 2002).

Integrando o tema medicamentos, o sangue, seus componentes e derivados são reconhecidos como recursos terapêuticos essenciais à saúde pública e também constam do debate nacional e internacional, em razão dos distintos interesses que os circundam, da regulamentação e fiscalização do seu suprimento e uso, por serem recursos necessários ao funcionamento dos sistemas de saúde. Assim, constituem objeto de política nacional em diversos países, conforme recomenda a Organização Mundial de Saúde. Cabe, portanto, o acompanhamento, a reflexão e a análise do processo de implantação e desenvolvimento das respectivas políticas públicas. No Brasil, a Política Nacional de Sangue, Componentes e Hemoderivados, publicada em 2001, para sua implementação demanda, organização, vigilância, uso racional, além do comprometimento de gestores, profissionais e sociedade civil, condições que se alinham às diretrizes de outras políticas de saúde, como a PNM.

Medicamentos, incluindo sangue e seus produtos, assistência farmacêutica e vigilância sanitária constituem um dos doze eixos temáticos do Observatório de Análise Política em Saúde (OAPS). A fim de estimular a reflexão e a análise crítica das políticas de saúde no Brasil, o OAPS surge como um espaço que envolve uma rede de pesquisadores da área de Política, Planejamento e Gestão em Saúde (PPGS) de diversos centros de pesquisa da área da saúde coletiva e afins para a produção de conhecimento crítico na área de Políticas de Saúde (OAPS, 2018; COSTA et al., 2018). O eixo Políticas de Medicamentos, Assistência Farmacêutica e Vigilância Sanitária propõe acompanhar a 
atuação do Estado brasileiro, os fatos produzidos pelo Ministério da Saúde e a Agência Nacional de Vigilância Sanitária (Anvisa) e as produções do Legislativo Federal, bem como o posicionamento de atores políticos envolvidos na temática. Parte-se do entendimento de que essas políticas são fundamentais para a garantia do direito à saúde e que o acesso aos medicamentos integra a atenção à saúde, envolvendo uma cadeia de fatores e uma complexa rede de atores públicos e privados com seus diferentes papéis e interesses. Tal dinâmica motivou a sistematização de procedimentos para análise dessas políticas, com base nas posições e ações de diferentes atores.

Assim, questiona-se como o Estado tem atuado e/ou se posicionado no que tange às políticas de Medicamentos e de Assistência Farmacêutica no período de 2014 à 2018 ? Delineia-se como objetivo: apresentar os resultados da análise da atuação do Estado brasileiro em torno das referidas políticas, no período de 2014 a 2018, tendo em vista o uso de estratégias de pesquisa utilizadas a partir de notícias de sites institucionais.

\section{Metodologia}

Trata-se de uma pesquisa longitudinal, realizada entre os anos 2014 a 2018, tendo como marco referencial os seguintes elementos: a ação do Estado em relação às políticas farmacêuticas; o medicamento e suas dimensões analíticas; a regulação sanitária de medicamentos; sangue e seus produtos, a análise política em saúde.

A construção dos dados deste estudo se deu com base na seleção de informações disponíveis em sítios eletrônicos de instituições, tomadas como atores no processo de implementação de ações relativas ao tema, com capacidade para se posicionar e/ou interferir na produção de fatos que implicam no ordenamento político-administrativo brasileiro. Foram consultados sítios eletrônicos das seguintes instituições: Ministério da Saúde (MS), Agência Nacional de Vigilância Sanitária (Anvisa), Conselho Nacional de Secretários de Saúde (Conass), Conselho Nacional de Secretários Municipais de Saúde (Conasems), Conselho Nacional de Saúde (CNS), Centro Brasileiro de Estudos em Saúde (Cebes) e Associação Brasileira de Saúde Coletiva (Abrasco). Também foram consultados sítios dos conselhos profissionais de Farmácia e de Medicina (CFM e CFF), a revista Radis-Fiocruz e a produção do Legislativo Federal.

Priorizou-se a busca de informações a partir do link notícias em cada um dos referidos sítios eletrônicos, por entender que neste estariam situadas as informações consideradas mais relevantes. Além disso, outros links dos sites foram visitados visando 
melhor qualificar a informação. Foram utilizados os descritores medicamentos, assistência farmacêutica, sangue e hemoderivados. O termo "vigilância sanitária" não foi incluído, já que este compreende diversificados objetos. Ponderou-se que a busca de notícias utilizando este descritor agregado aos outros resultaria em perda, dado que são mais restritas as notícias divulgadas que abordem as questões de vigilância sanitária envolvidas nos fatos relativos a medicamentos, assistência farmacêutica, sangue e seus produtos.

As informações sobre ações políticas do Estado, nos poderes Executivo e Legislativo, relacionadas a medicamentos e assistência farmacêutica, assim como os posicionamentos de atores institucionais a respeito de alguma ação do Estado foram consideradas relevantes para a análise. As notícias foram organizadas em planilhas padronizadas e individualizadas par cada sítio de consulta, contendo as seguintes informações: data da publicação da notícia, tema (se relativa a medicamentos, assistência farmacêutica, vigilância sanitária, sangue e hemoderivados), síntese/pauta (resumo da notícia), link de acesso, fonte e observações (comentários adicionais a respeito da notícia). A atividade de coleta das notícias propriamente dita foi feita mensalmente por bolsistas estudantes da graduação em farmácia e Saúde Coletiva - das instituições de Ensino Superior, Universidade do Estado da Bahia e Universidade Federal da Bahia.

Após a coleta e organização dos dados nas planilhas, pelos bolsistas, ocorria à validação pelo grupo de professores pesquisadores. Essa etapa foi realizada com periodicidade mensal, quando se discutia a relevância da informação e os seus desdobramentos, na perspectiva da análise política em saúde. Ou seja, o grupo se ateve à seleção dos fatos mais importantes que se apresentavam com possibilidades de se constituírem em fato político. Neste caso, tomou-se como referencial a discussão sobre fatos sociais, históricos e políticos produzida pelo OAPS (ALENCAR, 2016).

Após a validação, as planilhas foram disponibilizadas em ambiente virtual do Moodle para que fossem acessadas por todos os integrantes do grupo de pesquisa. As notícias também foram salvas, na íntegra, em formato PDF (Portable Document Format) e dispostas no ambiente virtual para evitar perdas face à possibilidade de sua exclusão dos sítios originais, por problemas no site, na configuração de linkages, entre outros.

Os dados sistematizados e validados foram organizados, para posterior análise, em quatro categorias analíticas, definidas pelo marco referencial que orientou os trabalhos dos doze eixos do OAPS: implementação da política, financiamento, participação social e resultados da política. Com base nesta estratégia metodológica o trabalho deste eixo de 
pesquisa do OAPS resultou em três produções: análise das políticas farmacêuticas tendo em vista a construção de uma análise política; análise das notícias segundo as diretrizes estruturantes da Política Nacional de Medicamentos (BRASIL, 1998); e a construção de uma linha do tempo (timeline) do eixo com os principais fatos, conforme as seguintes categorias: cenário internacional, cenário nacional, poder executivo, poder legislativo, poder judiciário, marco Jurídico-institucional, eventos, financiamento, gestão e posicionamento dos atores. Este artigo apresenta os resultados das duas primeiras referidas produções do eixo.

Essas produções foram desenvolvidas anualmente, de 2014 a 2018, e publicadas no sítio do OAPS ${ }^{7}$, na forma de Matriz de Acompanhamento (monitoramento anual das políticas de que tratam o eixo de acordo com os quatro categorias analíticas acima referidas, buscando-se correlacionar a posição - ação ou omissão - dos atores); Debates e Pensamentos (ensaios analíticos sobre um fato político específico relativo ao tema); e na caracterização do próprio eixo temático, no qual encontra-se disponível a linha do tempo.

De acordo com Esperidião (2018), na área da saúde diferenciam-se duas abordagens centrais de análise política: a 'Análise Política em Saúde' e a 'Análise de Políticas de Saúde'. A primeira inclui os estudos que tomam como objeto central a análise das relações de poder em saúde (natureza, estrutura, relações, distribuição e lutas) nos âmbitos setoriais e societário, ou o processo político em saúde, assim como suas relações com a produção de fatos políticos, dentro e fora das instituições; incluem estudos que examinam conjunturas e suas relações com a saúde. Já a segunda abordagem analítica agrupa os estudos sobre 'o conteúdo' das políticas enquanto diretrizes, planos e programas.

A análise política em saúde e a análise dos eixos estruturantes da Política Nacional de Medicamentos, realizadas nesse estudo tiveram como foco o processo político em saúde relacionado às políticas farmacêuticas do Brasil. Assume-se, nesta análise, o poder como categoria de análise central, ou seja, buscou-se identificar como ele é apropriado, acumulado, distribuído, disputado e utilizado nos âmbitos setorial e societário, assim como as suas relações com a produção de fatos, dentro e fora das instituições (OAPS, 2018).

\footnotetext{
${ }^{7}$ http://www.analisepoliticaemsaude.org
} 


\section{Resultados}

\subsection{Políticas farmacêuticas: análise dos principais fatos produzidos entre 2014 e 2018}

A análise dos dados relativos ao ano de 2014 permitiu entender que, no tocante à implementação de políticas, houve ampliação da cobertura, tanto no que se refere à disponibilidade de medicamentos, conforme Mengue et al., (2016) e Oliveira et al., (2016), resultados da Pesquisa Nacional sobre Acesso, Utilização e Uso Racional de Medicamentos - PNAUM Inquérito, quanto no fortalecimento dos serviços farmacêuticos, especialmente em função do Programa de Qualificação da Assistência Farmacêutica (QualifarSUS), como apontam os resultados da PNAUM Serviços (AKERMAN; FREITAS, 2017). Ocorreu também o fortalecimento das Parcerias de Desenvolvimento Produtivo (PDP) e a consolidação do respectivo marco regulatório estratégia do governo brasileiro para favorecer o desenvolvimento do Complexo Industrial da Saúde e a ampliação das tecnologias em saúde utilizadas no SUS.

Em relação ao financiamento, enfatiza-se que os gastos totais com medicamentos foram correlativamente superiores aos gastos totais em saúde, impactados de modo expressivo pelas ações judiciais para acesso a medicamentos. Outros investimentos para a qualificação dos serviços farmacêuticos também foram dispendidos.

O fenômeno da judicialização, que começou a se intensificar no início do ano 2000, caracteriza-se atualmente por mais de um milhão de ações, de modo que os gestores e as demais autoridades envolvidas com a saúde e a justiça não sabem o que fazer (SANTOS, 2018b). A autora alerta que na reflexão sobre o SUS deve-se pensar sobre os altos custos das tecnologias em saúde que nem sempre são necessárias nem representam verdadeiras inovações e acrescenta que "é preciso controlar o interesse no lucro dado que as indústrias farmacêuticas não são altruístas nem visam o bem-estar das pessoas e sim o lucro que podem alcançar" (p. 2049).

No que tange à categoria participação social, salienta-se a realização, em 2014, de oficinas de avaliação dos 10 anos da Política Nacional de Assistência Farmacêutica (PNAF), em 15 estados brasileiros, para subsidiar os debates da $15^{\text {a }}$ Conferência Nacional de Saúde. Registra-se, nesse período, conflitos importantes em torno da regulação sanitária de medicamentos anorexígenos que evidenciaram interferências do poder Legislativo na regulação desses produtos, em que pese a função legalmente estabelecida da Anvisa. O caso envolvendo os anorexígenos explicitou a intensa pressão que 
segmentos empresariais e parlamentares podem realizar sobre o Estado para atender situações pontuais e interesses corporativos e, em muitos casos tais divergências extrapolam as negociações entre os agentes reguladores estatais e o segmento regulado e passam a transitar no Judiciário e no Legislativo na forma de contestação e litigância (SILVA; COSTA; LUCCHESE, 2018, p. 1958).

Frise-se ainda a participação de diferentes atores sociais para a promulgação da Lei $\mathrm{n}^{\circ}$ 13.021, que dispõe sobre o exercício e fiscalização das atividades farmacêuticas, resultante de longo processo de debate entre empresários, parlamentares e a categoria farmacêutica. Encontravam-se em jogo os interesses da saúde pública versus os interesses do mercado e, conquanto pareça terem prevalecido os interesses públicos, fragilidades no processo fiscalizatório existentes no país podem implicar no predomínio dos interesses do capital no mercado farmacêutico (EMMERICK et al., 2015).

No referente ao ano de 2015, chamou atenção a incorporação de novos medicamentos, com possível ampliação da cobertura da assistência farmacêutica. Por outro lado, houve também desabastecimento de medicamentos essenciais, como a penicilina, justamente no momento em que houve aumento dos casos de sífilis no país, incluindo a sífilis congênita. Estratégias como acordos para compra conjunta de medicamentos foram utilizados para minimizar as consequências desse desabastecimento. Em relação ao financiamento, a tônica que foi o corte no orçamento da saúde.

No âmbito da regulação sanitária, a Lei nº 13.097/2015 trouxe mudanças para o trabalho da vigilância sanitária no Brasil, atualizando normas legais e os procedimentos de registro de medicamentos, produtos e equipamentos médicos, cosméticos e saneantes. Outro resultado importante refere-se à regulação dos produtos à base de Canabidiol em associação com outros canabinóides, por meio da RDC n ${ }^{\circ} 17 / 2015$, que possibilitou maior agilidade no processo de importação com regras específicas sobre o produto para pacientes que necessitem do tratamento. Vale ressaltar que tal regulamentação foi desencadeada pela participação social, representada por organizações da sociedade civil junto ao poder Legislativo e Executivo visando o atendimento dessa necessidade.

O ano de 2016 foi marcado por conflitos envolvendo o Legislativo e o Executivo no âmbito da regulação sanitária. A tentativa de regulação do uso da fosfoetanolamina substância utilizada em condições experimentais para tratamento de doenças oncológicas, mas sem a autorização da agência reguladora, pela falta de evidências de eficácia e segurança - e a retomada do debate sobre os medicamentos anorexígenos, por meio de 
Projetos de Lei do Legislativo contrário à posição da Anvisa, constituiu um fato relevante, por evidenciar interferências na autonomia da agência reguladora face a potenciais riscos à saúde. O caso da fosfoetanolamina sintética, entre outros, foram destaques emblemáticos de litigância contra regulações da Anvisa, e, portanto, caracterizam a relação tensa, e em geral conflituosa, entre os agentes públicos reguladores e os poderes Legislativo e Judiciário no Brasil, onde, como referem Silva, Costa e Lucchese (2018, p. 1958-1959), "nem sempre prevalecem argumentos de proteção da saúde e que retardam ou obstruem a concretização do direito à saúde, previsto na Constituição Federal".

Nesta perspectiva, cabe a discussão sobre a complexidade da regulação sanitária por sua atuação em funções muito sensíveis aos interesses econômicos, a exemplo do controle do tabagismo, dos preços dos medicamentos, de sua eficácia e segurança. Os conflitos decorrentes da mediação entre os interesses dos segmentos econômicos e as políticas públicas de proteção da saúde evidenciam possíveis repercussões negativas para a saúde da população, quando a regulação sanitária é realizada apenas com parâmetros da economia de mercado. Isto requer o aperfeiçoamento das políticas regulatórias que necessitam do engajamento de outros órgãos públicos para além do setor saúde, na formulação de políticas de alcance sócio sanitário em que a preservação da saúde humana tenha centralidade em vez de interesses setoriais transversais defendidos pelos segmentos econômicos (SILVA; COSTA; LUCCHESE, 2018).

Neste âmbito da regulação sanitária, no ano de 2016, foram efetivados diversos acordos e projetos de cooperação internacional, com potencial para contribuir na consolidação da imagem institucional da Agência Nacional de Vigilância Sanitária no cenário internacional, bem como no aperfeiçoamento da capacidade regulatória.

No referente ao financiamento da saúde, em geral, houve destaque deste tema, principalmente diante do cenário do impeachment da presidente Dilma Rousseff, no qual pautas como redução de gastos públicos, subfinanciamento e a aprovação da PEC 95 tiveram grande repercussão. No tocante à judicialização da saúde, medidas foram adotadas com possibilidade de implicações no financiamento. Trata-se de um termo de cooperação entre o Ministério da Saúde e o Conselho Nacional de Justiça (CNJ) que pretende fornecer subsídios para aprimorar o conhecimento técnico dos magistrados para a solução das demandas. Um aspecto positivo se refere a investimento no setor de produção pública de medicamentos, incluindo aqueles para doenças raras e negligenciadas. Na perspectiva da implantação de políticas, deu-se ênfase na ampliação do acesso ao tratamento de HIV/Aids. 
A EC-95/2016 restringiu o cálculo das despesas federais primárias exclusivamente à correção da inflação do ano anterior, desconsidera o crescimento populacional, a incorporação de tecnologias na qualidade dos serviços, o aumento do número de idosos e o subfinanciamento, ignora as demais necessidades sanitárias nos próximos 20 anos, colocando em risco o direito à saúde, afrontando, portanto, os objetivos fundamentais da República (SANTOS, 2018a). Como ressalta Santos (2018b) “a saúde fiscal não pode ocasionar dano moral e físico; tampouco pode afetar apenas as políticas sociais, devendo ser discutido de modo amplo, transparente e com responsabilidade, qualquer medida fiscal que afete a vida das pessoas" (p. 2049-2050).

A despeito dos resultados positivos do Programa Farmácia Popular do Brasil (PFPB), apontados em estudos, deu-se redução de investimentos na modalidade Aqui Tem Farmácia Popular e acentuou-se o fechamento de farmácias da modalidade Rede Própria, culminando com a decisão, do poder Executivo, pela finalização dessa modalidade, o que gerou reação da sociedade civil (ALENCAR et al., 2018).

O Programa Farmácia Popular do Brasil (PFPB), cujo modelo com ênfase no consumo de medicamentos como promotor de acesso a medicamentos, pautado na parceria com o comércio farmacêutico como principal forma de consolidação e expansão, significou um retrocesso, pelo caráter centralizador do Ministério da Saúde (MS) na provisão de medicamentos. Autores apontam ainda que esta política despertou discussões sobre sua interlocução com o modelo do SUS, trouxe dúvidas a respeito de sua ação concorrencial ou complementar, além de maiores custos comparados aos cenários públicos estudados pelos autores e ainda possíveis questionamentos deste modelo em relação à integralidade, uma vez que não se identificam ações dirigidas ao uso apropriado de medicamentos, tampouco de acompanhamento farmacoterapêutico, entre outros aspectos (SILVA; CAETANO, 2015; ALENCAR et al., 2018).

Em 2017 um fato marcante para todas as ações e serviços de saúde do SUS, incluindo a assistência farmacêutica, refere-se à aprovação, pela Comissão Intergestores Tripartite (CIT), da Portaria $n^{\circ}$ 204/2017, que modifica as modalidades de transferência de recursos do Fundo Nacional de Saúde (FNS) para estados, Distrito Federal e municípios, unificando os blocos de financiamento em apenas duas modalidades: custeio e investimento, anteriormente seis blocos temáticos. A mudança faz parte do Projeto SUS Legal e atendeu uma antiga reivindicação dos gestores da área da saúde. Segundo o Ministério da Saúde, o objetivo do governo federal foi o de possibilitar ao gestor ter mais agilidade e eficiência na destinação dos recursos. A medida foi bem recebida por gestores 
estaduais e municipais da saúde contudo, o Conselho Nacional de Saúde (CNS) afirmou que, embora possibilite a desburocratização da utilização dos recursos do SUS e atenda a uma antiga reivindicação de muitos especialistas e gestores, seria necessária uma análise minuciosa da matéria, principalmente levando em consideração o atual processo de subfinanciamento do SUS, que privilegia recursos para a média e alta complexidade em detrimento da atenção básica (BRASIL, 2004).

Destacou-se ainda em 2017, importante medida adotada com possibilidade de impacto no preço dos medicamentos que se refere ao Banco de Preços em Saúde (BPS), uma ferramenta online, gratuita e de acesso aberto ao público, desenvolvida pelo governo federal. Todas as compras de medicamentos realizadas no país, para o abastecimento do SUS, terão seus preços registrados neste Banco. Estados, municípios e Distrito Federal passam a alimentar de forma obrigatória essa ferramenta, visando dar transparência ao uso dos recursos públicos e conhecimento dos preços praticados em todo o território nacional (MINISTÉRIO DA SAÚDE, 2017).

No contexto de retrocessos nas políticas sociais no governo Temer, em 2017 ocorreram significativas mudanças no Programa Farmácia Popular do Brasil (PFPB) que resultaram em restrições no acesso a medicamentos. Inicialmente a Comissão Intergestores Tripartite (CIT) decidiu pela interrupção do financiamento da modalidade rede própria, sob alegação de elevado custo administrativo. As unidades da rede própria foram extintas, restando apenas a rede credenciada do Aqui tem Farmácia Popular (ATFP), alvo de críticas e questionamentos pelo TCU, não esclarecidos pelo DAF/MS. Verificou-se ainda que o fechamento da modalidade rede própria do programa Farmácia Popular, alvo de críticas de organizações da sociedade civil (ALENCAR et al., 2018).

O ano de 2018 teve como destaques ações com o propósito de assegurar o uso racional de antimicrobianos (PROQUALIS, 2018c). No mês de janeiro, a Organização Mundial da Saúde (OMS) divulgou os primeiros dados de vigilância sobre resistência aos antimicrobianos e revelou altos níveis de resistência a uma série de infecções bacterianas graves em países de alta e de baixa renda. A Anvisa deu destaque ao Sistema Global de Vigilância de Resistência Antimicrobiana (GLASS), desenvolvido para apoiar o plano de ação global sobre resistência antimicrobiana (RAM), que promove e apoia uma abordagem padronizada para a coleta, análise e compartilhamento de dados apoiada pela OMS (OPAS, 2018).

Sinaliza-se a tramitação do Projeto de Lei $\mathrm{n}^{\circ}$ 9.482/2018, de autoria do deputado federal Ronaldo Martins (PRB/CE) para autorizar a venda de medicamentos isentos de 
prescrição em supermercados, o que provocou posicionamentos contrários do CNS que aprovou uma recomendação contra o PL, justificando que a medida representa um retrocesso em todas as políticas públicas da área da saúde, em especial as que tratam do uso racional de medicamentos (BRASIL, 2004).

Sobre as PDP, teve destaque o caso envolvendo o Centro de Desenvolvimento e Produção de Medicamentos Sintéticos que recebeu investimentos do governo federal para sua modernização. A unidade faz parte do Instituto de Tecnologia do Paraná (Tecpar). O parque tecnológico do Paraná receberá $\mathrm{R}$ \$ 35,9 milhões para ampliar a produção de medicamentos; além deste recurso, terá $\mathrm{R} \$ 82$ milhões para a construção de uma nova unidade de fabricação e armazenamento de imunobiológicos em Maringá - PR (MINISTÉRIO DA SAÚDE, 2018a, 2018b).

A análise do Tribunal de Contas da União (TCU) sobre o possível favorecimento da TECPAR teve início em julho de 2018. Entre os pontos investigados foram citados a parceria com o laboratório paranaense, realizada sem o aval de órgãos colegiados e em desacordo com a Consultoria Jurídica do Ministério da Saúde. Em dezembro, o TCU decidiu abrir uma auditoria para apurar um contrato de $\mathrm{R} \$ 222,9$ milhões assinado pelo MS e pelo Tecpar. O contrato incluía o fornecimento de Trastuzumabe, medicamento usado no tratamento de câncer. O TCU afirmou que o preço cobrado pela fornecedora estava acima do praticado no mercado e determinou a suspensão do contrato de produção de medicamentos e a retenção de $27,39 \%$ de todas as despesas pagas e programadas para aquisição do medicamento. Segundo o TCU, o laboratório Bio-Manguinhos e o Instituto Butantan ofereceram preços menores para o referido medicamento: $\mathrm{R} \$ 892,60$ e $\mathrm{R} \$ 939,93$, respectivamente, o que provocou questionamento do ministro relator ao MS sobre os motivos da escolha da Tecpar como parceira na PDP, uma vez que o rito adotado parece ter limitado a possibilidade de outros laboratórios públicos serem credenciados para a parceria.

Segundo apurações do TCU e da mídia, a Tecpar é uma empresa do Governo do Paraná, estado governado à época pela esposa do então ministro da saúde Ricardo Barros. Documentos mostram a participação ativa de um ex assessor da Tecpar que foi para o MS na gestão do então Ministro, assumiu um cargo na Secretaria de Ciência e Tecnologia e Insumos Estratégicos e foi nomeado diretor do departamento do Complexo Industrial e Inovação em Saúde do Ministério da Saúde. Em um memorando, o secretário descreveu o novo formato das PDP de anticorpos monoclonais. Em uma nota técnica, de fevereiro desse ano, atesta-se o cumprimento de todos os requisitos da PDP, sinalizando as condições para o início de uma nova fase da produção. Neste documento, o preço do 
produto também é citado. Em 2017, o servidor citado retornou à Tecpar depois de atuar na negociação e assumiu o cargo de diretor industrial.

Ainda no tema das PDP, em agosto de 2018 foi noticiado pelo MS que a Empresa Brasileira de Hemoderivados e Biotecnologia (Hemobrás) irá receber do Governo Federal e de parceiros privados cerca de $\mathrm{R} \$ 642,9$ milhões para concluir a fábrica de fracionamento de plasma em Goiana - PE. O recurso é oriundo de uma nova parceria tecnológica firmada entre a Hemobrás/Ministério da Saúde e Tecpar/Octapharma (MINISTÉRIO DA SAÚDE, 2018c).

No referente ao Programa Farmácia Popular do Brasil, noticiou-se que o Tribunal de Contas de União (TCU) vai analisar o fechamento da rede própria deste programa. O órgão já investiga possíveis fraudes e custos acima do normal nos convênios estabelecidos pelo Ministério da Saúde com farmácias privadas que caracterizam a outra modalidade do programa, conhecida como Aqui tem Farmácia Popular. O Ministério da Saúde alegou que as fraudes causaram prejuízos de $\mathrm{R} \$ 500$ milhões anuais. (RADIS, 2018). Com a mudança, restou apenas a vertente do programa voltada para os convênios com as farmácias privadas, que oferecem medicamentos com descontos de até 90\%. O Cebes defendeu o programa e fez uma análise sobre o acesso público a medicamentos (CEBES, 2018).

Em 2018, chamou a atenção o caso da licitação para compra, pelo MS, dos medicamentos para doenças raras, Soliris, Fabrazyme, Myosyme e Aldurazyme. O processo para aquisição desses medicamentos foi paralisado por determinação do Ministério Público Federal, contrariando o ministro da saúde. O caso ganhou destaque na mídia porque a empresa vencedora da licitação não dispunha da licença de importação, dado que não cumpria uma norma da Anvisa que proíbe distribuidoras de comercializar medicamentos no Brasil quando não disponham de aval do respectivo laboratório produtor e a referida distribuidora, além de não estar autorizada a importar e comercializar tais medicamentos já havia recebido do MS um adiantamento no valor de mais de 19 milhões. O ministro da Saúde acionou representantes dos pacientes, órgãos de controle, Justiça, Advocacia Geral da União e parlamentares para acompanhar o caso (MINISTÉRIO DA SAÚDE, 2018d).

Em maio de 2018, representantes da cadeia de produção e distribuição de medicamentos para a o SUS discutiram propostas de melhorias para a assistência farmacêutica pública. Os participantes puderam expor gargalos da fabricação nacional de medicamentos para o SUS. O presidente do CONASEMS destacou os problemas gerados 
pela falta de interesse da indústria farmacêutica na produção de medicamentos de baixo custo. "Uma lista de itens que são importantes para milhões de usuários do SUS, mas não são atrativos para a indústria tem a fabricação descontinuada, os municípios não conseguem concluir processos licitatórios por falta de oferta e acabam ficando desabastecidos, isso gera um impacto enorme no SUS.

Outros resultados relativos ao Programa Aqui tem Farmácia Popular (ATFP), em 2018, dizem respeito ao estabelecimento de novos critérios de acesso aos medicamentos. O MS procedeu revisão dos valores repassados às farmácias credenciadas sob o argumento de sobrepreço de medicamentos e noticiou que estudos realizados no âmbito interno demonstraram que o preço da Insulina no ATFP era 2,5 vezes mais elevado que na rede pública e ainda que foram constatadas diferenças de preços em outros itens, tendose iniciado negociações com a indústria farmacêutica e com o setor varejista para debater o sobrepreço identificado (ALENCAR et al., 2018).

O ministro da saúde declarou que a revisão teve o objetivo de minimizar fraudes e que representaria economia de $\mathrm{R} \$ 750$ milhões, o custo do programa passaria de $\mathrm{R} \$ 2,6$ bilhões para R $\$ 1,85$ bilhão, reduzindo o custo total do programa, porém atendendo o mesmo número de brasileiros. Esta redução de investimento provocou conflitos com o mercado farmacêutico. A Associação Brasileira de Redes de Farmácias e Drogarias (Abrafarma) emitiu um comunicado público questionando o MS e afirmando que as reduções de preços deixariam seus custos a descoberto. Em abril de 2018, entrou em vigor a Portaria MS n 739, que atualizou os preços dos medicamentos do ATFP, com redução média de $38 \%$ do preço pago aos varejistas. Tal fato gerou pressão política do setor, que alegou que os preços definidos pelo MS inviabilizariam o programa, prejudicando o acesso da população aos medicamentos (ALENCAR et al., 2018).

\subsection{Análise dos fatos políticos à luz das diretrizes da Política Nacional de Medicamentos}

A análise dos fatos políticos relacionados a medicamentos, assistência farmacêutica e vigilância sanitária, produzidos no período de estudo, e do posicionamento dos atores políticos face às diretrizes estruturantes da PNM revelou variações de ênfases nas diretrizes nos dois espaços institucionais do poder executivo - MS e Anvisa - e nos distintos anos do período de estudo. Ademais, demonstrou poucos posicionamentos dos atores políticos que foram acompanhados. 
As notícias divulgadas pelo MS enfatizaram principalmente as três diretrizes da PNM, a saber: Acesso a medicamentos, Desenvolvimento cientifico e tecnológico, Reorientação da assistência farmacêutica com variações nos anos de estudo. No tocante às notícias divulgadas pela Anvisa as diretrizes mais enfatizadas foram a Regulação sanitária de medicamentos mais outras medidas relacionadas à qualidade, eficácia e segurança dos medicamentos; à promoção do uso racional e ao acesso a medicamentos, igualmente com variações entre os anos de estudo.

No tocante ao acesso a medicamentos, observou-se um esforço do poder executivo, com a incorporação de medicamentos novos, principalmente para favorecer o acesso da população a medicamentos para doenças raras e doenças de alta prevalência no país, a exemplo da Hepatite C e HIV/AIDS. Por outro lado, o Programa Farmácia Popular do Brasil que contribuiu para a ampliação do acesso a medicamentos essenciais foi parcialmente extinto em 2016, no bojo das medidas de restrição de investimentos em políticas sociais iniciadas no governo de Michel Temer. Diante da atual conjuntura econômica e política, se agravam os desafios para manter e ampliar o acesso a medicamentos; medidas de restrição de acesso podem ampliar padrões de desigualdade social.

Observou-se um esforço do poder executivo direcionado ao fortalecimento das Parcerias de Desenvolvimento Produtivo (PDP). Essa diretriz estabelece as estratégias para favorecer a produção de medicamentos e a ciência e tecnologia no país. No entanto, as PDP foram fortemente ameaçadas com a nova política econômica que restringe os investimentos do Estado - Emenda Constitucional 94/2016. Além disso, investimentos para o Programa de Apoio ao Desenvolvimento do Complexo Industrial da Saúde (Profarma), via BNDES foram colocadas em suspeição pela operação "Lava Jato". Após o impedimento da presidente Dilma Roussef, o Ministério da Saúde reconfigurou em 2016 a produção de anticorpos monoclonais pelos laboratórios públicos, incluindo a Tecpar como um dos parceiros principais, sem haver contudo atendimento às regras das PDP. Tal fato culminou em 2018 em suspensão da PDP com a Tecpar por determinação do TCU.

A assistência farmacêutica contou com mudanças relevante no financiamento dirigida a reorientação do modelo com destaque para o para Programa de Qualificação da Assistência Farmacêutica (Qualifar SUS) orientado para a estruturação das farmácias públicas e capacitação de pessoal. Merece referir a importância de ações do MS no sentido de organizar serviços farmacêuticos clínicos na atenção básica do SUS com o objetivo de 
identificar, prevenir e solucionar problemas relacionados ao uso de medicamentos pelos usuários.

As notícias divulgadas pela Anvisa enfatizaram principalmente a diretriz Regulamentação Sanitária mais um conjunto de ações direcionadas à garantia da qualidade, eficácia e segurança dos medicamentos, como seria esperado da instituição regulatória. Observou-se ênfase também na diretriz da promoção do uso racional e do acesso a medicamentos. Além disso, percebeu-se esforços do poder do executivo, através da Anvisa para interferir no controle de preços dos medicamentos. Entretanto, após o processo de Impeachment da Presidente Dilma Rousssef, importantes mudanças ocorreram em 2016 no governo Temer com a promulgação de uma Medida Provisória mudando as regras de atualização de preços de medicamentos. Tal medida provocou grande instabilidade jurídica e as opiniões tanto do controle social quanto do segmento regulado foram negativas resultando na perda da vigência desta MP em 2017. Acrescentese que, ainda no âmbito da regulação econômica dos medicamentos, deu-se a criação e implantação de uma plataforma para aquisição conjunta de medicamentos entre os países do Mercosul, cujo processo foi iniciado em 2015 e concretizado em 2017 com a realização da primeira compra conjunta; ademais de iniciativas para modernização e ampliação do sistema de regulação e monitoramento de medicamentos, em consonância/convergência com os marcos regulatórios internacionais.

\section{Considerações finais}

Os resultados deste estudo oferecem subsídios para a análise das políticas farmacêuticas, tendo-se encontrado nas conjunturas elementos relacionados ao poder em suas relações com a saúde, que favoreceram ou dificultaram a implementação de políticas farmacêuticas e regulatórias relevantes para a saúde da população brasileira.

Em que pesem os avanços, permanecem os desafios decorrentes do subfinanciamento público para a saúde, que impacta diretamente na capacidade de universalização e integralidade das ações e no acesso a medicamentos, e a permanente ameaça à continuidade das políticas de medicamentos, assistência farmacêutica e vigilância sanitária. Ademais, persiste o desafio de fortalecimento da regulação e vigilância sanitária, face aos conflitos de interesses que permeiam as relações entre os diversos atores da área e a necessidade contínua da atuação do Estado no seu papel de regulador do mercado de produtos e serviços relacionados à saúde, de modo a preservar 
os interesses da saúde coletiva e não a supremacia do mercado. Além disso, permanece a necessidade da mobilização e envolvimento da saciedade civil na defesa dos seus legítimos interesses na questão da saúde, em particular o direito ao acesso a medicamentos de qualidade, com eficácia e segurança e à assistência farmacêutica como parte da integralidade da atenção à saúde.

A estratégia de pesquisa adotada, neste estudo, possibilitou a construção de um espaço democrático e amplo para a elaboração de um pensamento crítico a respeito da saúde e dos processos políticos relacionados que se desenvolvem nas diferentes conjunturas. Este espaço manteve-se aberto à criatividade e permitiu a interação entre pesquisadores, docentes, graduandos, mestrandos e doutorandos, tornando possível a articulação de saberes e uma produção técnica articulada, que poderá favorecer o estabelecimento de redes de colaboradores interinstitucionais que trabalham os temas do eixo.

Salienta-se, ainda, que a estratégia metodológica utilizada possibilitou discutir e elaborar mecanismos para o estudo de políticas com base em fonte secundária, essencialmente a mídia eletrônica. Ao eleger como fonte os sítios eletrônicos dos principais atores coletivos foi possível fazer a articulação necessária para situar o posicionamento desses atores a respeito de um fato específico, bem como o confronto das informações encontradas. Frisa-se ainda a produção de diferentes produtos técnicos e científicos (ensaios, teses, dissertações, trabalhos de conclusão de curso de graduação e especialização em formato de residência), ampliando, dessa forma, as estratégias para debate a respeito do tema.

Por outro lado, o fato de ter trabalhado com várias fontes pode ter sido um fator limitante para o estudo. Isso porque a coleta de dados precisou ser feita por várias pessoas $\mathrm{e}$, ainda que todas estivessem cientes a respeito dos objetivos e do referencial orientador do eixo temático, a seleção das notícias pode ter sido influenciada pelo entendimento de cada um a respeito do conteúdo trazido. Assim, é possível que outras informações também importantes não tenham sido trabalhadas pelo grupo ao sistematizar os dados. Dito isto, permanece como pauta a necessidade de aprofundar o debate sobre o tema dos medicamentos, assistência farmacêutica e vigilância sanitária.

Por fim, há que se fortalecer o papel das agências reguladoras, não sucumbindo aos falsos argumentos sobre extrapolação de competência das mesmas, mas tendo clareza sobre a interferência e desequilíbrio que vem se dando entre as esferas do poder público, com destaque para o fortalecimento da prerrogativa de proteção da saúde da Anvisa, visto 
que a harmonia e a independência dos poderes, são elementos fundantes da Constituição (SILVA; COSTA; LUCCHESE, 2018, p. 1958).

\section{Referências}

ALENCAR, T. Notas sobre definições de fato social, histórico e político: subsídios para análise política em saúde. Observatório de Análise Política em Saúde, Bahia, p. 1-14, 2016. Disponível em: https://www.analisepoliticaemsaude.org/oaps/documentos/pensamentos/147428574757dfd0b3e 7102/. Acesso em: 12 jul. 2019

ALENCAR, T. et al. Programa Farmácia Popular do Brasil: uma análise política de sua origem, seus desdobramentos e inflexões. Saúde em Debate, Rio de Janeiro, v. 42, n. esp. 2, p. 159172, out. 2018.

ARAÚJO, P. et al. Pharmaceutical care in Brazil's primary health care. Revista de Saúde Pública, São Paulo, v. 51, p. 1-11, nov. 2017. Available from: https://doi.org/10.11606/S15188787.2017051007109. Access on: 10 july. 2019.

AKERMAN, M.; FREITAS, O. Pesquisa Nacional sobre Acesso, Utilização e Promoção do Uso Racional de Medicamentos (PNAUM): avaliação dos serviços de atenção farmacêutica primária. Revista de Saúde Pública, v. 51, p. 1-5, nov. 2017. Disponível em: https://doi.org/10.11606/S1518-8787.201705100supl2ed. Acesso em: 10 jul. 2019.

BARROS, J. A.C. Antigas e novas questões: enfrentando uma conjuntura desfavorável. In: BARROS, J. A. C. (org.). Os fármacos na atualidade antigos e novos desafios. 1. ed. Brasília: Anvisa, 2008a. p. 24-78.

BARROS, J. A. C. Nuevas tendencias de la medicalización. Ciênc. Saúde Coletiva, Rio de Janeiro, v. 13, n 4, p. 579-587, abr. 2008. Disponible:

http://www.scielo.br/scielo.php?script=sci_arttext\&pid=S141381232008000700007\&lng=en\&n rm=iso. Acceso en: 14 jul. 2019.

BERMUDEZ, J.A.Z.; BARROS, M. B. A. Perfil do acesso e da utilização de medicamentos da população brasileira - contribuições e desafios da PNAUM - Inquérito Domiciliar. Rev. Saúde Pública, São Paulo, v. 50, p. 1-4, 2016. Disponível em:

http://www.scielo.br/scielo.php?script=sci_arttext\&pid=S0034-

89102016000300201\&lng=en\&nrm=iso. Acesso em:14 jul. 2019.

BRASIL. Ministério da Saúde. Portaria no 3.916, de 30 de outubro de 1998. Aprova a Política Nacional de Medicamentos. Brasília, DF, 1998. Disponível em: http://www.saude.gov.br. Acesso em: 30 mar. 2010.

BRASIL. Presidência da República. Lei $\mathbf{n}^{0}$ 9.787, de 10 de fevereiro de 1999. Dispõe sobre a vigilância sanitária, estabelece o medicamento genérico, dispõe sobre a utilização de nomes genéricos em produtos farmacêuticos e dá outras providências. Brasília, DF, 1999a. Disponível em: http://www.anvisa.gov.br/e-legis. Acesso em: 30 mar. 2010.

BRASIL. Ministério da Saúde. Lei no 9.782 de 26 de janeiro de 1999. Define o Sistema Nacional de Vigilância Sanitária, cria a Agência Nacional de Vigilância Sanitária, e dá outras providências. Brasília, DF, 1999b. Disponível em: http://www.anvisa.gov.br/e-legis. Acesso em: 30 mar. 2012. 
BRASIL. Ministério da Saúde. Conselho Nacional de Saúde. Resolução no 338, de 06 de maio de 2004. Aprova a Política Nacional de Assistência Farmacêutica. Brasília-DF, 2004.

Disponível em: http://portal.saude.gov.br. Acesso em: 20 mar. 2010.

CEBES. Nota: Farmácia Popular e o acesso público a medicamentos, 2018. Disponível em: http://cebes.org.br/2018/05/nota-farmacia-popular-e-o-acesso-publico-a-medicamentos/. Acesso em: 07 de ago. 2018.

COSTA, E. A. et al. Technical issues and conservation conditions of medicines in the primary health care of the Brazilian Unified Health System. Rev. Saúde Pública, São Paulo, v. 51, p. 112, 2017. Available from: http://dx.doi.org/10.11606/s1518-8787.2017051007106. Access on: 09 july. 2019

COSTA, E. et al. Observatório de Análise Política em Saúde: Estratégias do Eixo Políticas de Medicamentos, Assistência Farmacêutica e Vigilância Sanitária para produção de conhecimento sobre o tema. In: CONGRESSO IBERO-AMERICANO EM INVESTIGAÇÃO

QUALITATIVA, 7., 2018. Fortaleza. Atas... Fortaleza: Ludomedia, 2018. p. 859-867.

Disponível em: https://proceedings.ciaiq.org/index.php/ciaiq2018/article/view/1856. Acesso em: 12 jul. 2019.

EMMERICK, I. C. M. et al. Farmácia Popular Program: changes in geographic accessibility of medicines during ten years of a medicine subsidy policy in Brazil. Journal of Pharmaceutical Policy and Practice, [S.I.], v. 8, n. 1, 10, mar. 2015. Available from: https://joppp.biomedcentral.com/track/pdf/10.1186/s40545-015-0030-x. Access on: 12 july. 2019.

ESPERIDIÃO, M. Análise política em saúde: síntese das abordagens teórico-metodológicas Political analysis in health: synthesis of theoretical-methodological approaches. Saúde Debate, Rio de Janeiro, v. 42, número especial 2, p. 341-360, out. 2018.

LEFÈVRE, F. O medicamento como mercadoria simbólica. 1. ed. São Paulo: Cortez Editora, 1991.

LUIZA V. L. Acesso à Assistência Farmacêutica: a relação dos setores público e privado de atenção à saúde. Riopharma, Rio de Janeiro, v. 11, n. 52, p. 4, nov./dez. 2002.

MENGUE, S. S. et al. Acesso e uso de medicamentos para hipertensão arterial no Brasil. FSP/USP. Rev Saúde Pública, São Paulo, v. 50, p. 1-9, 2016.

MINISTÉRIO DA SAÚDE. Agência da Saúde. Ministério da Saúde torna obrigatório registro de compras de medicamentos adquiridos para SUS. Disponível em: http://portalms.saude.gov.br/noticias/agencia-saude/28837. Acesso em: 12 fev. 2018.

MINISTÉRIO DA SAÚDE.TECPAR em Ponta Grossa terá R\$ 35,9 milhões para ampliar produção de medicamentos. Disponível em: http://portalms.saude.gov.br/noticias/agenciasaude/42890-tecpar-em-ponta-grossatera-r-35-9-milhoes-para-ampliar-producao-demedicamentos. Acesso em: 07 ago. 2018.

MINISTÉRIO DA SAÚDE. TECPAR terá R\$ 82 milhões para ampliar produção de medicamentos biológicos. Disponível em: http://portalms.saude.gov.br/noticias/agenciasaude/42734-tecpar-tera-r-82-milhoespara-ampliar-producao-de-medicamentos-biologicos. Acesso em: 07 ago. 2018.

MINISTÉRIO DA SAÚDE. Governo Federal anuncia R\$ 642,9 milhões para conclusão de fábrica da Hemobrás em Goiana (PE). Disponível em: 
http://portalms.saude.gov.br/noticias/agencia-saude/42881-governo-federal-anunciar-642-9milhoes-para-conclusao-de-fabrica-da-hemobras-em-goiana-pe. Acesso em: 07 ago. 2018.

MINISTÉRIO DA SAÚDE. Liminar aponta "afronta a concorrência" em compra para doenças raras, 2018d. Disponível em: http://www.saude.gov.br/noticias/agencia-saude/42669liminar-aponta-afronta-a-concorrencia-em-compra-para-doencas-raras. Acesso em: 08 ago. 2018.

MORGAN, S. G.; BOOTHE, K. Universal prescription drug coverage in Canada: Longpromised yet undelivered. Healthc Manage Forum, v. 29, n. 6, p. 247-254, nov. 2016 Available from: https://www.ncbi.nlm.nih.gov/pubmed/27744279. Access on: 10 mar. 2018.

OAPS. Observatório de análise política em saúde. Eixos temáticos. Ano 2015. Disponível em: http://www.analisepoliticaemsaude.org/oaps/eixos/. Acesso em: 01 mar. 2018.

OLIVEIRA, M. A. et al. Acesso a medicamentos para doenças crônicas no Brasil: uma abordagem multidimensional. Rev Saúde Pública, São Paulo, v. 50, p. 1-13, 2016. Disponível em: https://dx.doi.org/10.1590/s1518-8787.2016050006161. Acesso em: 5 out. 2018.

PAIM, J. S. Sistema Único de Saúde (SUS) aos 30 anos. Ciência \& Saúde Coletiva, Rio de Janeiro, v. 23, n. 6, p. 1723-1728. Jun. 2018. Disponível em: https://dx.doi.org/10.1590/141381232018236.09172018. Acesso em: 20 de nov. 2018.

PIGNARRE, P. O que é o medicamento? Um objeto estranho entre ciência, mercado e sociedade. 1. ed. São Paulo: Ed. 34, 1999.

RADIS. TCU investiga fim do Farmácia Popular, 2018. Disponível em: http://www6.ensp.fiocruz.br/radis/revista-radis/186/sumula/tcu-investiga-fim-do-farmaciapopular. Acesso em: 07 de ago. 2018.

RIBEIRO, G. S. Problemas de saúde da população brasileira e seus determinantes. In: PAIM, J. S.; ALMEIDA FILHO, N. Saúde Coletiva: Teoria e Prática. 1. ed. Rio de Janeiro: Medbook, 2014. p. 97-119.

SANTOS, N. SUS 30 anos: o início, a caminhada e o rumo. Ciência \& Saúde Coletiva, Rio de Janeiro, v. 23, n. 6, p. 1729-1736, jun. 2018a Disponível em: https://dx.doi.org/10.1590/141381232018236.06092018. Acesso em: 20 de out. 2018.

SANTOS, L. SUS-30 anos: um balanço incômodo? Ciência \& Saúde Coletiva, Rio de Janeiro, v. 23, n. 6, p. 2043-2050, jun. 2018. Disponível em: http://dx.doi.org/10.1590/141381232018236.06082018. Acesso em: 20 de out. 2018.

SILVA, J. A. A.; COSTA, E. A.; LUCCHESE, G. SUS 30 anos: Vigilância Sanitária. Ciência \& Saúde Coletiva, Rio de Janeiro, v. 23, n. 6, p. 1953-1962, jun. 2018. Disponível em: https://dx.doi.org/10.1590/1413-81232018236.04972018. Acesso em: 10 janeiro 2019.

SILVA, R.M.; CAETANO, R. "Farmácia Popular do Brasil" Program: characterization and evolution between 2004 and 2012. Cien Saude Colet, Rio de Janeiro, v. 20, n. 10, p. 29432956. oct. 2015.

SOUZA. G. et al. Characterization of the institutionalization of pharmaceutical services in Brazilian primary health care. Rev. Saúde Pública, São Paulo, v.51, p. 1-12 2017. Available from: http://dx.doi.org/10.11606/s1518-8787.2017051007135. Access on: 18 apr. 2018. 
VIACAVA, F. et al. SUS: oferta, acesso e utilização de serviços de saúde nos últimos 30 anos. Ciênc. saúde coletiva, Rio de Janeiro, v. 23, n. 6, p. 1751-1762, 2018. Disponível em: http://dx.doi.org/10.1590/1413-81232018236.06022018. Acesso em: 10 nov. 2018.

Recebido em: 22 de fevereiro de 2019.

Aceito em: 15 de julho de 2019. 